\title{
COMPARE 2013: Constraining tropical ocean cooling during the Last Glacial Maximum
}

\author{
David W. Lea' ${ }^{1}$ M. Kienast ${ }^{2}$, T. de Garidel-Thoron ${ }^{3}$, M. Kageyama ${ }^{4}$, A. Paul ${ }^{5}$ and E. Bard ${ }^{3}$
}

Corvallis, USA, 3 December 2013

Establishing a sea surface temperature (SST) distribution for the Last Glacial Maximum (LGM) has long been a primary goal in paleoclimate research, with the CLIMAP (Climate Long range Investigation, Mapping and Prediction) reconstruction standing out as a landmark accomplishment. The development of new SST proxies such as UK37' and Mg/Ca paleothermometry in the 1980s and 90s spurred the international community to re-evaluate LGM SSTs. The publication of the SST reconstruction of MARGO (Multiproxy Approach for the Reconstruction of the Glacial Ocean surface) was the culmination of an international collaboration to update and improve LGM SST reconstructions (MARGO Project Members 2009). The MARGO reconstruction is an important validation target for the modeling community because the climate forcing and response during the LGM are relatively well known and allow for an assessment of climate sensitivity determinations.

The purpose of the COMPARE 2013 workshop was to follow up on an initial meeting of COMPARE 2012 (Comparing Ocean Models with Paleo-Archives) in Bremen, Germany (18-21 March 2012) which had discussed ways to further improve the MARGO data set and how to apply it to data-model intercomparisons (Kucera et al. 2012). The MARGO SST reconstruction synthesizes both more traditional approaches (i.e. faunal transfer functions) and newer geochemical approaches (i.e. Mg/Ca and UK37'). But because the original MARGO data set for the tropics is still dominated by estimates based on transfer functions, the reconstructed SST changes do not optimally represent the different proxy results. The COMPARE 2013 workshop brought together observationalists and modelers to exchange their latest insights on tropical SST proxies and the efficacy of the MARGO low latitude reconstructions.
There are many reasons for re-evaluating LGM tropical SST, including the MARGO compilation itself, which displays a much stronger level of spatial heterogeneity in the tropics than has been simulated by coupled model runs (Kageyama et al. 2013); the validity of the SST proxies and their overall compatibility; advances in linking climate sensitivity to LGM tropical cooling in models (Hargreaves et al. 2012); downward revisions of LGM cooling based on MARGO; and advances in analyzing and attributing deglacial SST records, which are increasingly based on geochemical proxy records (Shakun et al. 2012).

We identified two overarching research questions to frame the problem:

\section{- Can we reconcile UK37' and Mg/Ca data within acceptable brackets by using harmo- nized calibration schemes and by consider- ing differences in habitat depth and season? If so, what was the magnitude of mean annual LGM tropical surface cooling?}

- How much heterogeneity was there in tropical surface cooling during the LGM?

To address these questions, we propose to develop a new LGM tropical SST geochemical database. This database will focus on measured parameters: i.e. raw Mg/Ca and UK37' measurements (Fig. 1). It will include the full data sequence from 0-30 ka BP with chronological constraints and uncertainties, associated parameters such as oxygen isotopes, alkenone abundance, dissolution parameters, $\mathrm{Mg} / \mathrm{Ca}$ on other species, and relevant metadata (e.g. how the samples were processed or cleaned, foraminiferal size fraction and morphotypes).

The proposed approach will provide an SST field using harmonized calibrations, which will allow direct comparison of $\mathrm{Mg} / \mathrm{Ca}$ and alkenone temperatures. It will also provide a basis of comparison with the MARGO (2009) database as well as a target for modeling. Statistical analysis of the dataset, considering the interproxy difference, the spatial reproduc ibility, as well as the calibration uncertainty, will provide uncertainty estimates for the SST field. The raw measurements can also be adapted to improved or alternative calibrations, data assimilation or forward modeling. Furthermore, assembling a database with records covering the full sequence since the last glacial opens the possibility of developing time slices for target periods other than the LGM (e.g. Heinrich Stadial 1 or the Younger Dryas).

We will try to implement a dynamic database approach that allows for the addition of new data as they become available, and, possibly, implementation of different calibration schemes. We invite members of the paleoceanographic community to submit UK37' and Mg/Ca data sets that will enhance the COMPARE effort.

\section{AFFILIATIONS}

'Department of Earth Science, University of California, Santa Barbara, USA

'Department of Oceanography, Dalhousie University, Halifax, Canada

${ }^{3}$ CEREGE, Aix-Marseille Université, Aix-en-Provence, France

${ }^{4}$ LSCE/IPSL, Gif-sur-Yvette, France

${ }^{5}$ MARUM - Center for Marine Environmental Sciences, University of Bremen, Germany

CONTACT

David Lea: lea@geol.ucsb.edu

\section{REFERENCES}

Hargreaves JC et al (2012) Geophys Res Lett 39, doi: 10.1029/2012GL053872

Kageyama M et al. (2012) Clim Dyn 40: 2469-2495

Kucera M et al. (2012) PAGES news 20(2): 102

MARGO Project Members (2009) Nat Geosci 2: 127-132

Shakun JD et al. (2012) Nature 484: 49-54
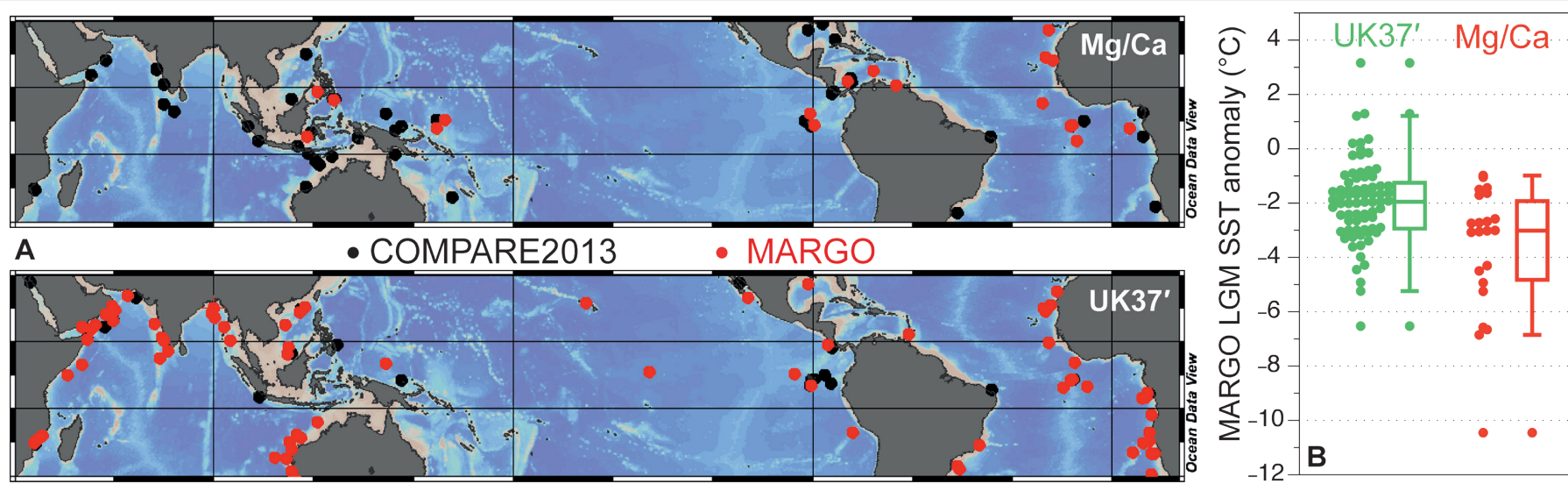

Figure 1: (A) Distribution of newly available low latitude records (black dots) compared to the MARGO (2009) dataset (red dots) that have Mg/Ca (top panel) and UK37' (lower panel) paleothermometry data. Note that sites are concentrated along oceanic margins. (B) Whisker plot comparison of low latitude UK37' and Mg/Ca data compiled in MARGO (2009). On average, $\mathrm{Mg} / \mathrm{Ca}$ data indicates $3^{\circ} \mathrm{C}$ of tropical cooling vs. $2^{\circ} \mathrm{C}$ for UK37'. 